\title{
Percepción del comensal sobre los restaurantes del programa Outstanding Host en la Av. Revolución de Tijuana
}

\author{
Perception of the diner about the \\ restaurants of the Outstanding Host program \\ at Av. Revolución de Tijuana
}

Enrique Rangel Rodríguez ${ }^{1}$, Margarita Ramírez Ramírez²

Resumen: El sector turistíco es uno de los motores más importantes en México, ya que es parte esencial de la economía nacional, a través de la generación de empleos, inversiones y derrama económica. Derivado de ello, la Secretaría de Turismo de Baja California (SECTURE) diseñó el programa de calidad Outstanding Host, el cual se implementó con la finalidad de brindar mayor certidumbre a las personas que visitaban la región, a través de establecimientos que cumplieran con la oferta de servicios y productos de calidad. De esta manera, este primer ejercicio tiene como objetivo conocer la percepción de los comensales sobre el mismo, y determinar si los restaurantes que forman parte del programa ubicados en la Av. Revolución de Tijuana, B.C., han cumplido con las características requeridas para ser miembros de él.

Palabras clave: Percepción, turismo, Outstanding Host.
Abstract: The tourism sector is one of the most important engines in Mexico, since it's an essential part of the national economy through employment generation, investment and economic impact. Therefore, the Ministry of Tourism of Baja California (SECTURE) designed the quality program Outstanding Host, which was implemented with the objective of providing more certainty to people who visited the region through establishments that provide them quality in products and services. Thus, this exercise aims to know the perception of the customers about the program, and determine if the restaurants that are part of it, located in Av. Revolucion in Tijuana, B.C., have complied with the characteristics required for being members.

Key words: Perception, tourism, Oustanding Host.

(Presentado: 5 de noviembre de 2017. Aceptado: 20 de diciembre de 2017)

\footnotetext{
${ }^{1}$ Licenciado en Mercadotecnia por la Universidad Autónoma de Baja California, México. Email: erangelquabc.edu.mx

${ }^{2}$ Doctor en Educación, Profesora Investigadora en Universidad Autónoma de Baja California, México. Email: mramirezquabc.edu.mx
} 


\section{INTRODUCCIÓN}

Como consecuencia de las modificaciones que se han dado en los patrones de consumo en los turistas a nivel internacional y nacional, en donde los viajeros buscan destinos que les ofrezcan mayor certidumbre y seguridad, se volvió necesaria la creación del programa Outstanding Host para los visitantes.

Este programa tiene como objetivo principal poder brindar mayor certidumbre a los visitantes que acuden a establecimientos que ofertan algún producto o servicio en Baja California. Esto a través de la distinción de los negocios que cumplen con calidad en la proporción de productos y servicios, dentro del programa se encuentran los rubros: alimentos, bebidas, hospedaje y otros.

Derivado de lo anterior, este trabajo se enfocará en la percepción de los comensales sobre los alimentos y bebidas, toda vez que por la naturaleza del producto, se torna de vital importancia contar con la certeza de que los establecimientos que se visitan cumplan con características de calidad.

En este sentido, (Johns y Pine, 2002) indican que los factores fundamentales que contribuyen a la satisfacción del cliente en un restaurante son: el alimento (higiene, nutrición), la distribución física (Layout, mobiliario y limpieza), el ambiente (confort), y la atención (amabilidad, velocidad y preocupación).

Al cumplir con estos aspectos, los establecimientos automáticamente pueden ser elegibles para ser parte del programa, perspectiva que les otorga a ellos una diferenciación del resto y coadyuva a la buena imagen de los mismos.

La mejora constante de la calidad de servicio es una condición indispensable para cualquier organización cuyo interés resida en prosperar (Giménez y Prior, 2004).

La combinación de lo anterior con la calidad en los productos, provoca que se vuelvan aspectos que coadyuvan a la existencia de establecimientos que brindan certidumbre y confianza a los comensales. Es por este motivo, que conocer la percepción de los comensales sobre los establecimientos que son parte del programa, será de gran ayuda para conocer la imagen que tienen de los mismos y definir estrategias que pudieran requerirse, con el objetivo de continuar ofertando calidad en productos y servicios.

El presente documento muestra un avance del proyecto "Percepción del comensal sobre los restaurantes del programa Outstanding Host de la SECTURE. El Caso de la Av. Revolución en Tijuana", en el que se observa la primera y segunda fase, misma que comprende fundamentos teóricos del trabajo de investigación, la metodología utilizada y resultados preliminares.

\section{REVISION LITERARIA}

Los comensales que reciben productos y servicios de calidad de un establecimiento determinado, generalmente forman una buena percepción sobre el mismo y como resultado prefieren visitarlos con mayor frecuencia, recomendarlos a sus seres queridos y hablar bien de los mismos.

Acorde a (Morris C. y Maisto A., 2011), las experiencias preceptúales, por lo regular, van más allá de la información sensorial disponible. En efecto, las experiencias perceptuales rara vez, si acaso, corresponden exactamente a la información que se recibe por medio de los sentidos. La percepción es una combinación de información de los sentidos, la experiencia previa y el cableado del encéfalo.

Todos los seres humanos normales tienen los mismos órganos sensoriales y capacidad perceptual. Sin embargo, la individualidad (motivaciones, valores, expectativas, estilo cognoscitivo e ideas culturales preconcebidas), influyen en lo que se percibe.

Para concluir y derivado de lo anterior, tal como lo mencionan (Morris C. y Maisto A., 2011), se generan percepciones, principalmente en base a los siguientes criterios:

- Motivación: Nuestros deseos y necesidades dan forma a nuestras percepciones. Es muy probable que la gente que tiene una necesidad perciba algo que piensa que podrá satisfacer esa necesidad.

- Valores: En un experimento que reveló con qué fuerza los valores de una persona afectan sus percepciones.

- Estilo cognoscitivo: Conforme maduramos, desarrollamos un estilo cognoscitivo (una forma personal de tratar 
con el ambiente) y esto también afecta la manera en que vemos el mundo. El estilo cognoscitivo también puede verse desde la perspectiva de los "igualadores" y los "exagerados", aquellos que nivelan las distinciones entre los objetivos y los que las magnifican.

- Experiencia y cultura: Los antecedentes culturales también influyen en las percepciones de la gente. El Lenguaje que la gente habla afecta la forma en que percibe su entorno. Las diferencias culturales en las experiencias de las personas también determinan como usan las señales perceptuales.

- Personalidad: Varios investigadores han demostrado que nuestra personalidad individual influye en nuestras percepciones. (Morris C. y Maisto A., 2011).

En el mismo orden de ideas, tal como lo indican (Robbins S. y Judge T., 2009), la percepción es el proceso por el que los individuos organizan e interpretan las impresiones de sus sentidos con objeto de asignar significado a su entorno. Sin embargo, lo que uno percibe llega a ser muy diferente de la realidad objetiva.

De esta manera, es importante mencionar que la percepción tiene un papel importante para este cometido, algunos factores operan para conformar y en ocasiones distorsionarla. Estos radican en el receptor, en el objeto percibido, y en el contexto de la situación en la que tiene lugar la percepción, como se muestra en la

\section{Gráfico $N^{\circ} 1$. Factores que influyen en la percepción.}

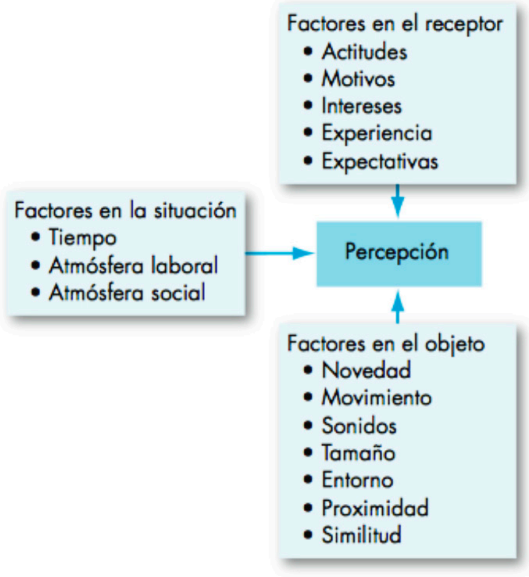

Fuente: (Robbins, S. y Judge, T., 2009).
Sobre las características del objeto que se observa afectan lo que se percibe. Como los objetos no se observan en forma aislada, la relación de un objeto con su entorno también influye en la percepción, así como en la tendencia a agrupar los objetos cercanos y similares.

Para concluir, acorde a (Robbins S. y Judge T., 2009), también es importante el contexto en el que se observan los objetos o eventos. El momento en que se miran influye en la atención, así como la ubicación, iluminación, calor o cualquier tipo de factor situacional.

Por lo tanto, analizar las expectativas y opiniones del servicio es elemento clave para determinar y gestionar la calidad del servicio prestado, por cuanto la misma (OMT 2006) indica que la calidad en el turismo implica la satisfacción de todas las necesidades, exigencias y expectativas de los clientes.

En el mismo orden de ideas, los autores (Kotler P. y Armstrong G., 2013), conciben a la calidad del servicio como la diferencia existente entre las expectativas y la percepción del servicio, y que para satisfacer a los clientes se deben conocer muy bien las expectativas para poder ofrecer un servicio de calidad, es decir, un servicio adaptado a sus expectativas.

Por otro lado, es importante tomar en cuenta que los errores durante diversos momentos de la interacción, o durante el proceso de prestación del servicio provocar que aparezcan sentimientos de inseguridad en el cliente quien dudará sobre la calidad del servicio recibido, volviéndolo vulnerable a las ofertas de la competencia y modificando la percepción que tenga sobre el lugar.

Lo anterior provocará que sus expectativas y zona de tolerancia, crezcan y se haga más reducida, respectivamente, tanto para los procesos (manera en que se presta el servicio), como para el resultado del servicio; es decir, el cliente será más impaciente si tiene que volver y se molestará con mayor facilidad.

De esta manera, al ser la calidad del servicio una variable de mucha importancia, se vuelve vital realizar un análisis, precisamente sobre las expectativas, así como la percepción experimentada por los clientes, para conocer la experiencia vivida posterior al servicio. 


\section{METODOLOGÍA}

Para (Hair, J., Bush, R., Ortinau, D., 2010), la muestra es un grupo de personas u objetos tomado de la población objetivo y existen dos diseños básicos de muestreo: el probabilístico y el no probabilístico. En el muestreo no probabilístico se desconoce la probabilidad de seleccionar cada unidad de muestreo. La selección de unidades de muestreo se basa en algún tipo de juicio intuitivo o conocimiento del investigador. De igual forma, dentro de los muestreos no probabilísticos se encuentra el muestreo por conveniencia, en el cual las muestras se extraen acorde a la comodidad.

Derivado de lo anterior y en base a la información proporcionada por la Secretaría de Turismo del Estado de Baja California, se seleccionaron 12 restaurantes que son parte del programa Outstanding Host en la Av. Revolución de Tijuana, B.C. Por lo tanto, con el objetivo de conocer la percepción que tienen los comensales sobre dichos establecimientos, la población meta definida se traduce en encuestar, a través de la conveniencia, a 100 comensales que hayan visitado y consumido en los establecimientos que son parte del programa al menos una vez.

Elaboración de herramientas para la obtención de datos:

\section{Descripción del instrumento}

Acorde a (Hernández-Sampieri, 2010), en toda investigación se aplica un instrumento para medir las variables contenidas en las hipótesis (y cuando no hay hipótesis, simplemente para medir las variables de interés). Esa medición es efectiva cuando el instrumento de recolección de los datos realmente representa las variables que se tienen en mente. Desde luego, no hay medición perfecta, es prácticamente imposible que se representen fielmente variables tales como la inteligencia, la motivación, el nivel socioeconómico, el liderazgo democrático, la actitud hacia el sexo y otras más.

Es importante mencionar que los cuestionarios pueden ser aplicados de distintas formas. Este en particular fue a través de entrevista personal, es decir, un entrevistador aplicó el cuestionario a los entrevistados, haciéndole las preguntas y anotando las respuestas.

\section{Encuesta (bloques de preguntas, Escala Likert)}

Para encontrar y conocer la percepción de los comensales sobre los restaurantes que forman parte del programa Outstanding Host en la Av. Revolución de Tijuana B.C., se diseñó una encuesta que permitiese obtener la información necesaria para cumplir con los objetivos del proyecto. Se realizó el diseño de una encuesta piloto para llegar a la encuesta final a aplicar.

Se utilizaron, principalmente, para este cuestionario preguntas con escala Likert, misma en las que se les solicita a las personas entrevistadas que elijan el grado de acuerdo o desacuerdo que tienen con una serie de enunciados de creencias mentales o conductuales sobre un determinado objeto, tema o situación.

En este mismo orden de ideas, para el proyecto se tomaron en cuenta las variables independientes: Imagen, servicio y calidad; y la dependiente percepción, acorde a los objetivos de la investigación y con el apoyo del diagrama Ex Ante como se observa a continuación:

Definición de variables

\section{Gráfico № 2. Definición de Variables.}

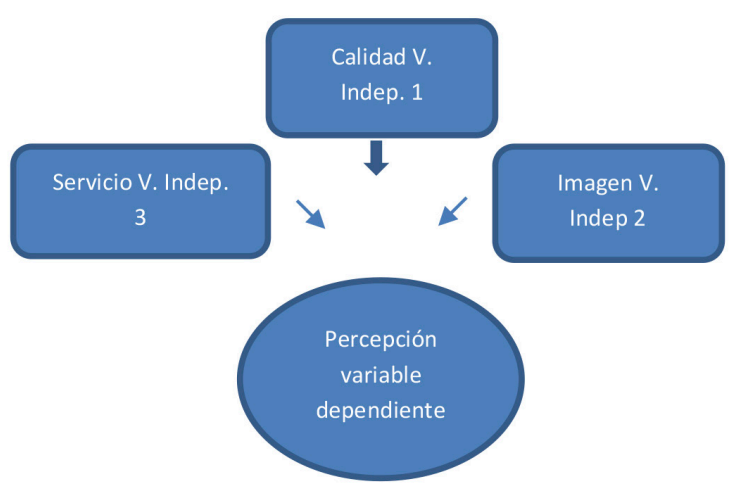

Fuente: (Elaboración propia, 2016).

\section{RESULTADOS PRELIMINARES}

Tabla N 1.- Estudios de confiabilidad - Alpha de Cronbach

\begin{tabular}{lcccc}
\hline No & Variable & N$^{\circ}$ de ítems & Factores & $\begin{array}{c}\text { Alpha de } \\
\text { Cronbach } \\
\text { Final final }\end{array}$ \\
\hline 1 & Percepción & 3 & 1 & .738 \\
2 & Imagen & 6 & 1 & .616 \\
3 & Calidad & 9 & 1 & .776 \\
4 & Servicio & 4 & 1 & .808 \\
\hline
\end{tabular}

Fuente: Elaboración propia, 2016. 
En la tabla se puede observar que al realizar el análisis de confiabilidad de los resultados de la aplicación del cuestionario, se obtienen elevados índices de Alfa de Cronbach, lo que indica que la confiabilidad del instrumento es significativa. También, se puede observar que la relación entre factores y Alfa de Cronbach indica que el instrumento tiene validez de contenido significativo.

\section{Análisis estadístico descriptivo}

Se lleva a cabo el análisis descriptivo de las variables correspondientes a la muestra de la investigación, esto con el fin de conocer las características de los comensales encuestados. Las gráficas y tablas describen las características principales de los comensales que participaron en esta investigación. Fueron un total de 100 percepción variable dependiente Servicio V. Indep. 3, Calidad V. Indep. 1, Imagen V. Indep. 2, comensales encuestados y a continuación se enlistan los datos más relevantes obtenidos relacionados a la variable percepción:

- Datos estadísticos de las variables.

- Frecuencia de presencia de las posibles respuestas.

- Gráfico explicativo de las respuestas obtenidas.

Para la aplicación de cuestionarios se contemplaron a comensales que hubieran visitado al menos una vez los establecimientos que forman parte del programa en la Av. Revolución de Tijuana, B.C.

Dichos restaurantes fueron seleccionados debido a su acreditación como parte del programa, siendo esta avenida una de las arterias principales de la ciudad y en donde prácticamente nació y se ha desarrollado el turismo desde principios del siglo XX.

Es importante mencionar que los cuestionarios fueron contestados por personas que se encontraban en el lugar y/o iban de salida, que ya habían comido o bebido algo. Esto de lunes a viernes, en horarios matutinos y vespertinos en un lapso de dos semanas.

\section{DISCUSIÓN}

Gráfico $N^{\circ} 3$. Un $67 \%$ de los comensales comentó que existía mucha relación entre el precio que pagaron y el servicio que les ofrecieron, un $26 \%$ extrema relación, mientras que un $7 \%$ indicó que tuvo poca.

\section{Relación entre el precio y el servicio $\mathrm{N}=100$}

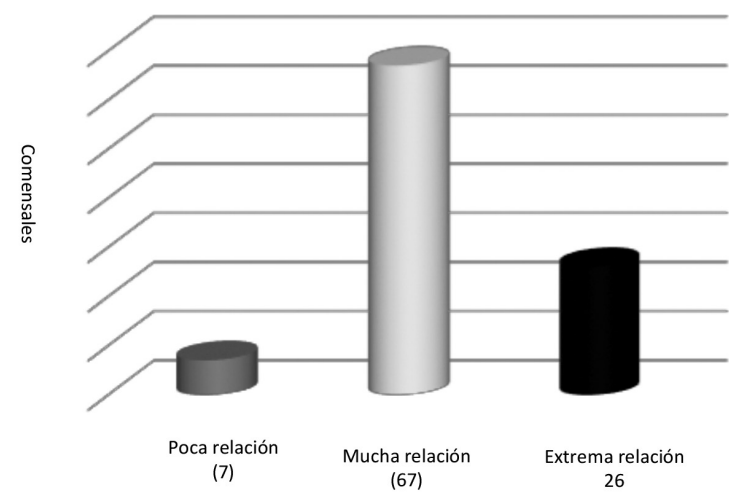

Fuente: Percepción del comensal sobre el programa Outstanding Host de la Secretaría de Turismo del Estado de Baja California. El caso de la Av. Revolución de Tijuana, UABC, 2016.

\section{Experiencia en el restaurante $\mathrm{N}=\mathbf{1 0 0}$}

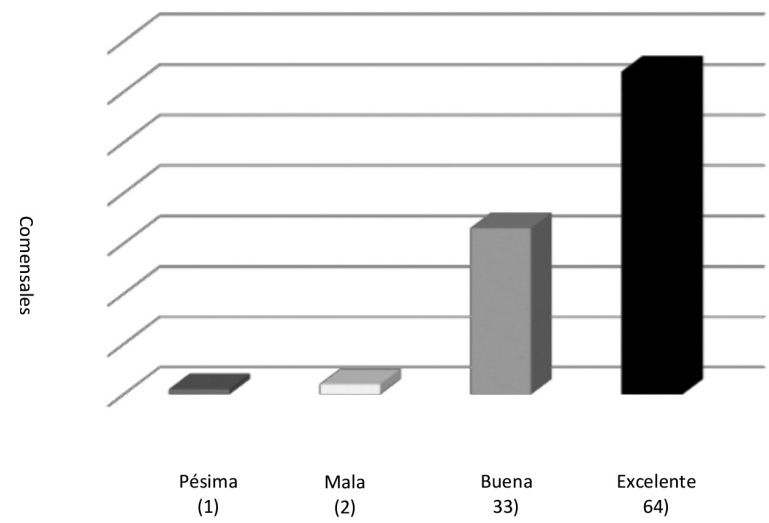

Fuente: "Percepción del comensal sobre el programa Outstanding Host de la Secretaría de Turismo del Estado de Baja California. El Caso de la Av. Revolución en Tijuana", UABC, 2016.

\section{CONCLUSIONES}

Derivado de lo anterior se puede concluir que uno de los grandes retos para el sector turístico, es la satisfacción del cliente, la calidad en el servicio y el crecimiento de la afluencia de visitantes.

Actualmente, los visitantes han dejado en segundo plano 
la cuestión económica al momento de acudir a un destino, en cambio ellos le han otorgado mayor relevancia al valor de vivir una experiencia inolvidable, respaldada por servicios y productos de calidad.

Para los países con vocación turística, estos son aspectos que se toman como base para el desarrollo de actividades, aprovechando sus ventajas competitivas tales como: Calidez y hospitalidad de los ciudadanos, atractivos turísticos e infraestructura.

Por lo tanto, el conocimiento de la percepción y las experiencias de los visitantes, se ha tornado en el sector en una actividad sumamente importante, ya que brinda la pauta para que las empresas y establecimientos conozcan los comentarios de sus usuarios sobre los servicios y productos ofertados. Y de esta manera puedan conocer sus fortalezas y áreas de oportunidad.

Este proyecto de investigación cuenta con un avance del $70 \%$, del cual se han desarrollado la primera y segunda fase, misma que comprende fundamentos teóricos del trabajo de investigación, la metodología utilizada y resultados preliminares.

\section{REFERENCIAS}

Giménez,V., Prior,D. (2004). Evaluación y fijación de objetivos en calidad de servicio. Un enfoque frontera. Revista Española de Investigación de Marketing, España.

Hair, J., Bush, R. y Ortinau, D. (2010). Investigación de Mercados. México, D.F.: McGraw-Hill.

Hernández-Sampieri, R. (2010). Metodología de la Investigación. México, D.F.: McGraw-Hill.

Johns, N. y Pine, R. (2002). Comportamiento del consumidor en la industria de la comida y servicio: Una revisión. Oxford, U.K: International Journal of Hospitality Management.

Kotler, P. y Armstrong, G. (2013). Fundamentos de Marketing. México, D.F.: Pearson Educación.

Morris, C. y Maisto, A. (2011). Introducción a la Psicología. México, D.F.: Pearson Educación.

Robbins, S. y Judge, T. (2009). Comportamiento Organizacional. México, D.F.: Pearson Educación. 